\title{
Torsades de Pointes: Unanswered Questions
}

\author{
Borys Surawicz \\ Krannert Institute of Cardiology, Indiana University School of Medicine, Indianapolis, Indiana
}

\begin{abstract}
Torsade de pointes (Tdp) is a polymorphic ventricular tachycardia (VT) in which the axis of the QRS complex changes direction after a certain number of complexes as if the complex rotated around the baseline. Tdp is usually associated with QT prolongation, and dispersion of ventricular repolarlization (DR). Experimental models of tdp are usually associated with induction of early after depolarizations (EADs). Several aspects of the pathogenesis of tdp are incompletely understood. The purpose of this article is to propose the directions in research that may increase our current understanding of the factors responsible for tdp.

The most plausible hypotheses requiring further supporting evidence are: 1 . The occurrence of tdp requires the presence of DR i.e. tdp does not occur in the absence of DR. 2. EADs appear to play an important role as a trigger to tdp in the animal models, but more evidence are needed at the clinical level. 3. EADs may be responsible for arrhythmias other than tdp. 4 . The greater incidence of tdp in the females than in tha males may be attributed to differences in the duration of the QT interval and different morphology of the ST-segment and the T-wave. The above gender differences may be caused by the effects of gonadal hormones which modulate some of the membrane currents flowing during early ventricular repolarization.
\end{abstract}

(J Nippon Med Sch 2002; 69: 218-223)

Key words: dispersion of repolarization, early after depolarizations, long QT, gender gap of repolarization

Torsade de pointes ( $\mathrm{Tdp}$ ) is a polymorphic ventricular tachycardia (VT) in which the axis of the QRS complex changes direction after a certain number of complexes as if the complex rotated around the baseline ${ }^{1}$. The phasic variation of the polarity and the amplitude of the QRS complexes may be apparent only if several synchronous leads are recorded. The intervals between complexes vary, and the rate of tachycardia ranges from 150 to 300 beats $/ \mathrm{min}^{2}$. In addition to its characteristic morphologic features, tdp differs from sustained monomorphic VT by the difficulty of arrhythmia induction with programmed electrical stimulation, and by the characteristic pattern of onset. In the majority of cases, tdp is preceded by a characteristic sequence of a long RR interval of the dominant cycle, followed by a short extrasystolic interval with premature depolarization interrupting the $\mathrm{T}$ wave (R on $\mathrm{T}$ phenomenon).

Many experimental studies suggest that tdp may be caused by a process known as early after depolarizations (EADs) in the presence of increased dispersion of repolarization (DR). The relative contribution of these two factors i. e. the assumed trigger of EAD and the underlying substrate of DR is difficult to establish in practice ${ }^{3}$. This is because it is seldom possible to assess these two phenomena simultaneously in the same preparation. The recognition

Correspondence to Borys Surawicz, M.D., M.A.C.C., 8333 Naab Rd, Suite 400. Indianapolis, IN 46260

E-mail: tscott@thecaregroup.com

Journal Website (http://www.nms.ac.jp/jnms/) 


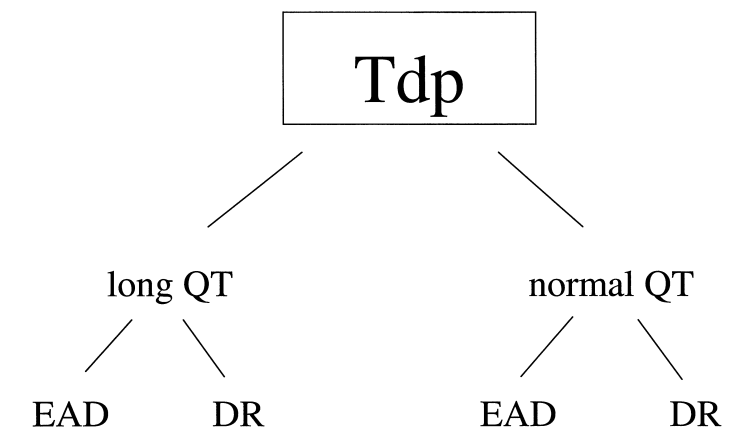

Fig. 1 Abbreviations: $\mathrm{Tdp}=$ torsade de pointes $\mathrm{EAD}=$ early after depolarizations $\mathrm{DR}=$ dispersion of repolarization

of $\mathrm{EAD}$ requires recording transmembrane action potentials in isolated tissues, or less reliably recording monophasic action potentials from ventricular surfaces whereas a reliable clinical assessment of DR requires recording from a large precordial area with a multitude of electrodes. In addition, EADs are generally easier to elicit in the Purkinje than in the ventricular muscle fibers which makes it difficult to recognize whether an $\mathrm{EAD}$ in the ventricular muscle is or is not transmitted from the Purkinje fibers. Moreover, the experimental conditions that are created to induce EADs tend to be nonphysiologic, and thus of questionable relevance to the conditions associated with tdp in the clinical setting. Finally, many of the polymorphic VTs recorded in the isolated tissues, or whole animal models merely resemble tdp, but do not conform to the definition of classical tdp.

Fig. 1 shows that tdp may be associated with either a prolonged or normal duration of the QT interval, and that in each case one assumes the possible contribution of the EAD and DR. Patients with normal QT interval in this category have usually no structural heart disease. Excluded from this scheme is polymorphic VT that may be morphologically indistinguishable from tdp, but occurs during myocardial ischemia, or in the presence of cardiomyopathy in which polymorphic VT coexists with other types of ventricular tachyarrhythmias.

The purpose of the subsequent discussion is to review our current understanding of the factors responsible for tdp by seeking answer to the following 4 questions :

1. Does tdp occur without increased DR?
2. Does tdp occur without EADs?

3. Can EADs produce no arrhythmias or arrhythmias other than tdp?

4. Why are women at greater risk of developing tdp than males?

\section{Association of tdp with increased DR.}

Dispersion of repolarization can result from one or more of the following: dispersion of ventricular action potential durations (APDs), dispersion of refractory periods which may or may not differ from the dispersion of APDs, and dispersion of activation times. It is reasonable to assume that dispersion at closely adjacent sites may be more disturbing than global dispersion of similar magnitude.

Both clinical and experimental observations suggest that tdp is associated with increased DR. In the clinical setting, tdp in the presence of long $\mathrm{QT}^{4-6}$, or normal $\mathrm{QT}^{7}$ is most often triggered by the $\mathrm{R}$ on $\mathrm{T}$ phenomenon. This phenomenon indicates the presence of increased DR because the dispersion must be equal to or greater than the interval between the onset of premature depolarization and the end of the $\mathrm{T}$ wave ${ }^{48}$.

In the study of 91 patients with pause-dependent tdp, relative QTc was one of the 3 independent predictors of ventricular fibrillation, the other 2 being low ventricular ejection fraction and presence of structural heart disease. However, QTc lengthening may be present without an apparent increase of the risk of tdp. The rare occurrence of tdp in patients with QT prolongation caused by treatment with amiodarone has been attributed to an absence of increased $\mathrm{DR}^{10.11}$. Recently, van Opstal et $\mathrm{al}^{12}$ compared the effects of chronic amiodarone and dronedarone in dogs with complete A-V block. Both drugs increased QTc similarly. Amiodarone did not increase significantly the difference between monophasic action potential durations (MAPD) and produced neither EADs nor ventricular ectopic activity whereas dronedarone increased the difference between MAPDs and produced both EADs and ectopic activity. Yan et $\mathrm{al}^{13}$ found that in the isolated ventricular preparations of rabbits, the $\mathrm{iKr}$ blocker d-sotalol produced QT prolongation, increased DR, EADs and ventricular arrhythmias resemling tdp. However, 
the drug azimilide which blocks both iKr and iKs produced an even greater QT prolongation and EADs but no increased DR and no ventricular tachyarrhythmia. It appears therefore that the occurrence of tdp requires the presence or increased DR, and that long QT interval in the absence of increased DR is not a definite risk factor for tdp.

\section{Does tdp occur in the absence of EADs?}

In the animal models of tdp, the presence of EADs was either manifest of could not be ruled out. A partial list of such models includes: dogs with chronic A-V block treated with class III antiarrhythmic drugs ${ }^{14}$, isolated rabbit hearts treated with quinidine in the presence of low $\mathrm{K}^{15}$, or with sotalol in the presence of bradycardia ${ }^{16}$, rabbits treated with azimilide and sotalol ${ }^{13}$, and dogs anesthetized with halothane and treated with sotalol ${ }^{17}$.

In the dog model of long QT syndrome (LQTS) induced by anthopleurin A, El Sherif et $\mathrm{al}^{18}$ recorded 3-dimensional maps using 256 bipolar electrograms during polymorphic VT with an apparent shift in QRS axis. In this preparation, arrhythmia was initiated by a subendocardial focal activity suspected to result from EADs.

EAD-like activity was recorded in patients with congenital LQTS ${ }^{19}$, and in those with drug-induced LQTS and $\operatorname{tdp}^{20}$. Gilmour et $\mathrm{al}^{21}$ studied patients with acquired LQTS and repeated episodes of tdp. They reported that the onset of tdp was precipitated by critical prolongation of QT interval, but not every long QT episode precipitated arrhythmia which suggested to the authors that the appearance of arrhythmias required presence of EADs. Thus, the bulk of the available evidence suggests, but does not prove that EADs play a critical role in the initiation of tdp. Lazzara ${ }^{22}$ accepted the evidence that the trigger of arrhythmias in the study of El Sherif et $\mathrm{al}^{18}$ has been identified as EADs, but pointed out that “... the mechanism for the maintenance of $\mathrm{VT}$ and its distinctive undulating pattern have remained mainly speculative". Restivo ${ }^{23}$ commented that “...today there is no compelling evidence that EAD-induced triggered activity arises in the intact heart and is the primary event that provokes the onset of tdp". In the opinion of El-Sherif ${ }^{24}$ : "Focal early afterdepolarization-induced triggered beat (s) can infringe on the underlying substrate of inhomogeneous repolarization to initiate polymorphic reentrant ventricular tachycardia that sometimes has a characteristic twisting of the QRS axis referred to as torsades de pointes." In vitro, the occurrence of EADs is not limited to Purkinje fibers. In the preparation of arterially perfused canine left ventricular wedge with a combined block of Kr and Ks currents, phase 2 EADs appeared in $\mathrm{M}$ and epicardial cells i.e. the two cell types with the greatest prolongation of action potential duration at slow rates ${ }^{25}$. Phase 3 EADs were not observed. In this preparation acceleration from an initially slow rete facilitated the development of $\mathrm{EADs}^{25}$. It appears, therefore, that EADs can be viewed as likely triggers of experimental arrhythmias associated with long QT interval, but the triggering role of EADs in the clinical setting requires more evidence.

\section{Can EADs produce no arrhythmias or arrhythmias other that tdp?}

Yan et $\mathrm{al}^{13}$ showed that in the models of acquired LQTS in $\operatorname{dog}$ and rabbit ventricular preparations, EADs often failed to propagate transmurally. This suggests that EADs can be present in the absence of manifest arrhythmias. There is also abundant evidence that EADs are often associated with isolated premature ventricular complexes and monomorphic VT in the absence of tdp. Thus, it can be concluded that there is no obligatory link between EADs and tdp.

\section{Increased prevalence of tdp in females}

The gender difference in the duration of the QT interval and the morphology of ventricular repolarization have been known for more than half century $^{26}$. More recently attention has been drawn to the gender difference in the incidence of tdp. The evidence for this is overwhelming, and I will highlight only few selected references. Among the first-or second degree relatives of patients with the congenital long QT syndrome, the females in the age group of $18^{-40}$ years, were at a higher risk of life-threatening cardiac events than the males ${ }^{27}$. Below age 18, however, the incidence of cardiac 
events in males and females was similar. In patients with acquired QT prolongation, women made up 70\% of the 332 reported cases of tdp related to the use of cardiovascular drugs ${ }^{28}$. Similarly, women made up $72 \%$ of cases of tdp in the setting of acquired complete atrio-ventricular block in the absence of drugs prologing the QT interval ${ }^{29}$. Of the reported 11 cases of tdp in association with probucol therapy ${ }^{30}$, all victims were women. In the review of the reported cases of tdp as a complication of subarachnoid hemorrhage, 12 of 19 subjects were women ${ }^{31}$. In my review of randomly collected 20 recent papers reporting cases of tdp caused by a variety of non antiarrhythmic drugs, which included antihistaminics and antibiotics, the ratio of women to men was 55 to $16^{26}$. It may be safely concluded that in all examined conditions of acquired QT prolongation, women developed tdp about 3 times more often than men, and that the occurrence of tdp was independent of the presence or absence of various clinical descriptors. It appears plausible that the longer QT interval and the female type of ventricular repolarization are in some way responsible for the female preponderance for the tdp.

Marked female preponderance has been reported also in hypothyroid patients with prolonged QT interval and recurrent ventricular tachycardia ${ }^{32}$ as well as in patients with global $\mathrm{T}$ wave inversion and prolonged QT interval in the absence of ischemic heart disease ${ }^{33}$. The latter condition, however, does not appear to be associated with tdp.

To find the cause of the gender difference in the incidence of tdp is a daunting task. The successful solution of the gender gap puzzle will probably require a collaborative effort of clinical investigators, physiologists, molecular biologists, geneticists, and biophysicists. The study cannot be confined to women because some men also are at risk of an excessive QT prolongation and tdp after an exposure to agents that prolong the QT interval. It has to be acknowledged that even though, the women are at a higher risk than men, the absolute number of subjects at risk of developing either an excessive QT lengthening, or tdp when exposed to customary doses of drugs that prolong the QT interval, is extremely small. This is illustrated by the study of Lehmann et $\mathrm{al}^{34}$ who examined records of 1,897 patients exposed to the iKr blocking drug d, l-sotalol without developing tdp. They found that at each dosing extreme the J-Tc interval, independent of the baseline JTc, was significantly longer in women than in men, but the difference was only in the range of 6-7 percent which is not sufficient to explain the gender gap in the incidence of tdp. This suggests that it will be more helpful to investigate the possible causes of predisposition to tdp, both in women and in men who develop tdp in the setting of excessive QT prolongation. It needs to be determined whether such subjects are less tolerant of bradycardia, perhaps as a result of an inherent abnormal QT/RR relation in which the QT values deviate from the normal values more at long than at short $R R$ intervals. Also, it needs to be established whether the risk of developing tdp is caused by an abnormal QT/RR relation that becomes manifest after exposure to agents that prolong the QT-interval, or cause hypokalemia. Of further interest may be to examine the effect of beta adrenergic agonists on the QT interval and the $\mathrm{QT} / \mathrm{RR}$ relation in the subjects prone to the development of the tdp. Selected subjects at risk for excessive QT prolongation, and/or development of tdp, will become candidates for appropriate genetic explorations.

The success of the studies may depend on the acquisition of the appropriate human myocytes for the study of membrane channels. The random selection of the gender representatives is not advisable because not all subjects in the general population have a very typical male or a very typical female repolarization patterns in the general population. The most fruitful approach may be to compare the subjects with the most contrasting patterns. The typical male pattern is easily recognizable by the higher takeoff of the $\mathrm{J}$ point and a steeper slope of early repolarization. Of these, the most distinct feature is the rapid onset of repolarization that is particularly prominent in the young men with the so called early repolarization pattern.

The available evidence points at the male hormones as the modifiers of ventricular repolarization after puberty. Lehmann et $\mathrm{al}^{35}$ expressed the view that we need to focus attention on the factors that 
shorten QT in men and protect against excessive lengthening of QT interval at slower heart rates that may be responsible for the development of tdp. It is known that the nonhuman primates have androgen receptors in both atrial and ventricular myocytes ${ }^{36}$. Dihydrotestosterone caused shortening of action potential at the level of 30 percent of repolarization in the papillary muscles of oophorectomized rabbits ${ }^{37}$. The reported effects of testosterone on human ECG are inconsistent. In one study treatment with intramuscularly administered hormone lessened post-exercise-induced ST-segment depression ${ }^{38}$, but in a more recent study intravenous administration of testosterone had no effect on the ECG in 20 men whose average age was 70.6 years $^{39}$.

\section{Conclusions}

Tdp is a fairly distinct ventricular tachyarrhythmia that is often self-terminating, but is capable of inducing syncope and sudden cardiac death. The principal approach to prevention and treatment is to avoid and correct precipitating factors. As an emergent treatment intravenous administration of magnesium salts is often effective as well as prompt increase of heart rate by atropine, isoproterenol, or pacing. The increase in heart rate may be expected to shorten QTc, decrease DR, and suppress EADs.

The available information reveals several gaps in our knowledge about the factors precipitating and sustaining tdp. These gaps relate to the substrate, trigger, their interrelation, and the predilection of female gender to tdp. How much better off will we be in dealing with the clinical aspects of tdp if we successfully eliminate the deficiencies in our knowledge about tdp? We know that improved knowledge of the arrhythmia mechanism does not always translate into an immediate improvement of prophylaxis and therapy in clinical practice. This may be also the case after unraveling some of the unanswered questions concerning tdp. Nevertheless, it cannot be disputed that each successive step toward improved understanding of a problem contributes to the scientific progress, the direction of which is not always anticipated. This includes the challenges posed by the unanswered questions about tdp.

\section{References}

1. Dessertenne T: La tachycardie ventriculaire a deux foyers variables. Arch Mal Coeur 1966; 59: 263-272.

2. Coumel P, Leclercq JF, Lucet V: Possible mechanisms of arrhythmias in the long QT syndrome. Eur Heart J $1985 ; 6$ : 115-129.

3. Surawicz B: Electrophysiologic substrate of torsade de pointes: Dispersion of repolarization or early afterdepolarizations? J Am Coll Cardiol 1989; 14: 172-184.

4. Surawicz B: Ventricular fibrillation and dispersion of repolarization. J Cardiovasc Electrophysiol 1997;

5. Locati EH, Maison-Blanche P, Dejode P, Cauchemez B, Coumel P: Spontaneous sequences of onset of torsade de pointes in patients with acquired prolonged repolarization. Quantitative analysis of Holter recordings. J Am Coll Cardiol 1995; 25: 1564-1575.

6. Viskin S, Alla SR, Barron HV, Heller K, Saxon L, Kitzis I, van Hare GF, Wong MJ, Lesh MD, Scheinmann MM: Mode of onset of torsades de pointes in congenital long QT syndrome. J Am Coll Cardiol 1996; 28: 1262-1268.

7. Leenhardt A, Glaser E, Burguera M, Nurnberg M, Maison-Blanche P, Coumel P: Short-coupled variant of torsade-de pointes: a new electrocardiographic entity in the spectrum of idiopathic ventricular tachyarrhythmia. Circulation 1994; 89: 206-215.

8. Svernhage E, Houltz B, Blomstroem P, Brachmann J, Crijns H, Jensen S, Vallin H, Swedberg K, Edvardsson N: Early electrocardiographic signs of drug-induced torsades de pointes A.N.E. 1998; 3 : 252-260.

9. Da Costa A, Chaldivan T, Belounas A, Messier M, Viallet M, Mansour H, Lamaison D, Dijane P, Isaaz K: Predictive factors of ventricular fibrillation triggered by pause-dependent torsades de pointes associated with acquired long QT interval: Role of QT dispersion and ventricular function. J Cardiovasc Electrophysiol 2000; 11: 990-997.

10. Cui G, Sen L, Sager PT, Uppal P, Singh BN: Effects of amiodarone, sematilide and sotalol on QT dispersion. Am J Cardiol 1995; 75: 465-469.

11. Hohnloser $\mathrm{S}$, Klingenheben $\mathrm{T}$, Singh $\mathrm{BN}$ : Amiodarone-associated proarrhythmic effects: a review with special reference to torsades de pointes tachycardia. Ann Intern Med 1994; 121: 529-533.

12. van Opstal JM, Schoenmakers M, Verduyn SC, de Groot M, Leunissen DJM, van der Hulst FF. Molenschot MMC, Wellens HJJ, Vos MA: Chronic amiodarone evokes no torsade de pointes arrhythmias despite QT lengthening in an animal model of acquired long-QT syndrome. Circulation 2001; 104: 2722-2727.

13. Yan G-X, Wu Y, Liu T, Wang J, Marinchak RA, Kowey PR: Phase 2 early afterdepolarization as a trigger of polymorphic ventricular tachycardia in acquired long-QT syndrome. Direct evidence from intracellular recordings in the intact left ventricular wall. Circulation 2001; 103: 2851-2856. 
14. Verduyn SC, Vos MA, van der Zande J, Kulcsar A, Wellens HJ: Further observations to elucidate the role of interventricular dispersion of repolarization and early afterdepolarizations in the genesis of acquired torsade de pointes arrhythmias. A comparison between almokalant and d-sotalol using the dog as its own control. J Am Coll Cardiol 1997; 30: 1578-1584.

15. Asano Y, Davidenko JM, Baxter WT, Gray RA, Jalife J: Optical mapping of drug-induced polymorphic arrhythmias and torsade de pointes in the isolated rabbit heart. J Am Coll Cardiol 1997; 29: 831-842.

16. Zabel M, Hohnloser SH, Behrens S, Li Y-G, Woosley RL, Franz MR: Electrophysiologic features of torsade de pointes: Insights from a new isolated rabbit heart model. J Cardiovasc Electrophysiol 1997; 8: 1148-1158.

17. Weissenburger J, Nesterenko VV, Antzelevitch C: Transmural heterogeneity of ventricular repolarization under baseline and long QT conditions in the canine heart in vivo: Torsades de pointes develops with halothane but not pentobarbital anesthesia. J Cardiovasc Electrophysiol 2001; 11: 290-304.

18. El-Sherif N, Chinushi M, Caref EB, Restivo M: Electrophysiological mechanism of the characteristics electrocardiographic morphology of torsade de pointes tachyarrhythmias in the long-QT syndrome. Detailed analysis of ventricular tridimensional activation patterns. Circulation 1997; 96: 4392-4309.

19. Shimizu W, One T, Kurita T, Takaki H, Aihara N, Kamakura S, Matsuhisa M, Shimomura K: Early afterdepolarizations Induced by isoproterenol in patietns with congenital long QT syndrome. Circulation 1991; 84: 1915-1923.

20. Kurita T, Ohe T, Shimizu W, Suyama K, Aihara N, Yakaki H, Kamakura S, Shimomura K: Early afterdepolarizationlike activity in patients with class IA induced long QT syndrome and torsades de pointes. Pacing and Clinical Electrophysiol 1997; 20: 695-705.

21. Gilmour RF, Riccio ML, Locati EH, Maison-Blanche P, Coumel P, Schwarz PJ: Time-and rate-dependent alterations of the QT interval precede the onset of torsade de pointes in patients with acquired QT prolongation. J Am Coll Cardiol 1997; 30: 209-217.

22. Lazzara R: Twisting of the points. J Am Coll Cardiol 1997; 29: 843-844.

23. Restivo M: Animal models of the long QT syndrome: Relation to clinical features. J Cardiovasc Electrophysiol 1997; 8 : 1148-1158.

24. El-Sherif N: Mechanism of ventricular arrhythmias in the long QT syndromes: On hermeneutics. J Cardiovasc Electrophysiol 2001; 12: 973-976.

25. Emori T, Antzelevitch C: Cellular basis for complex $\mathrm{T}$ waves and arrhythmic activity following combined Ikr and Iks block. J Cardiovasc Electrophysiol 2001; 12: 1369-1378.

26. Surawicz B: Puzzling gender repolarization gap. J Cardiovasc Electrophysiol 2001; 12: 613-615.
27. Zareba W, Moss AJ, Le Cessie D, Locati EH, Robinson JL, Hall WJ, Andrews MI: Risk of cardiac events in family members of patients with long QT syndrome. J Am Coll Cardiol 1995; 26: 1685-1691.

28. Makkar RR, Fromm BS, Steinmann RT, Meissner $\mathrm{MD}$, Lehmann MH: Female gender as a risk factor for torsades de pointes associated with cardiovascular drugs. JAMA 1993; 270: 2590-2597.

29. Kawasaki R, Machado C, Reinoehl Fromm B, Baga JJ, Steinmann RT, Lehmann MH J: Increased propensity of women to develop torsades de pointes during complete heart block. J Cardiovasc Electrophysiol 1995; 6 : 1032-1038.

30. Reinoehl J, Frankovich D, Machado C, Baga JJ, Meissner MD, Lehmann MH, Steinmann RT: Probucol-associated tachyarrhythmic events and QT prolongation: Importance of gender. Am Heart J 1996; 131: 1184-1191.

31. Machado C, Baga JJ, Kawasaki R, Reinoehl J, Steinmann RT, Lehmann MH: Torsade de pointes as a complication of subarachnoid hemorrhage. J Electrocardiol 1997; 30: 31-37.

32. Nesher G, Zion MM:Recurrent ventricular tachycardia in hypothyroidism. Report of a case and review of the literature. Cardiology 1988; 75: 301-306.

33. Desai SA, Mehrok S, Spodick DH: Global T-wave inversion: Limited QT dispersion despite QTc prolongation-a correlate of benignity in patients with strikingly abnormal electrocardiograms. Clin Cardiol 1999; 22: 655-657.

34. Lehmann MH, Hardy S, Archibald D, Mac Neil DJ: JTc prolongation with d, l-sotalol in women versus men. Am J Cardiol 1999; 83: 354-359.

35. Lehmann MH, Timothy KW, Frankovich D, et al: Age-gender influence on the rate-corrected QT interval and the QT-heart rate relation in families with genotypically characterized long QT syndrome. J Am Coll Cardiol 1997; 29: 93-99.

36. McGill HC Jr, Anselmo VC, Buchanan JM, Shendan PJ: The heart is a target organ for androgen. Science 1980; 207: 775-776.

37. Hara M, Danilo P Jr, Rosen MR: Effect of gonadal steroids on ventricular repolarization and on response to E 4031. J Pharmacol Exp Ther 1998; 285: 1068-1072.

38. Jaffe MD: Effect of Testosterone ciprionate on posterxercise ST segment depression Br Heart J 1977; 19: 1217-1222.

39. White CM, Ferraro-Borgida MJ, Moyna NM, et al: The effect of pharmacokinetically guided acute intravenous testosterone administration on electrocardiographic and blood pressure variables. J Clin Pharmacol 1999; 39: 1038-1043.

(Received, February 12, 2002)

(Accepted, February 20, 2002) 